\title{
Anurofauna (Amphibia, Anura) em um remanescente de Floresta Ombrófila Mista no Estado de Santa Catarina, Sul do Brasil
}

\author{
Elaine Maria Lucas $^{1,2}$ \& João Carlos Marocco ${ }^{1}$ \\ ${ }^{1}$ Área de Ciências Exatas e Ambientais, Universidade Comunitária da Região de Chapecó, Av. \\ Senador Atílio X. Fontana 591 E, Bairro Efapi, CEP 89809-000, Chapecó, SC, Brasil \\ ${ }^{2}$ Autor para correspondência: Elaine Maria Lucas, e-mail: elaine.lucasg@gmail.com
}

LUCAS, E.M. \& MAROCCO, J.C. Anurans (Amphibia, Anura) in a remnant of mixed ombrophilous forest in Santa Catarina State, Southern Brazil. Biota Neotrop. 11(1): http://www.biotaneotropica.org.br/v11n1/en/ abstract?article+bn01211012011.

\begin{abstract}
Faunal surveys are important tools to enhance biological conservation strategies in different regions. This study presents information on amphibian species richness and species composition in Parque Nacional das Araucárias; the largest conservation unit of mixed ombrophilous forest in Santa Catarina State, Southern Brazil. Active searches were conducted in October 2008 and March 2009 in sites potentially used for reproduction of species. We recorded a total of twenty nine species, being 28 native and one exotic species, and belonging to 16 genera and eight families. The high richness recorded, as well as the occurrence of species associated with nearly pristine habitats, suggest that Parque Nacional das Araucárias is an important area for amphibian conservation in Santa Catarina State.
\end{abstract}

Keywords: amphibians, species richness, conservation, Araucaria Forest.

LUCAS, E.M. \& MAROCCO, J.C. Anurofauna (Amphibia, Anura) em um remanescente de Floresta Ombrófila Mista no Estado de Santa Catarina, Sul do Brasil. Biota Neotrop. 11(1): http://www.biotaneotropica. org.br/v11n1/pt/abstract?article+bn01211012011.

Resumo: Inventários de fauna são importantes ferramentas para subsidiar estratégias para a conservação da diversidade biológica em diferentes regiões. Este estudo apresenta informações sobre a riqueza e a composição de espécies de anuros no Parque Nacional das Araucárias, a maior Unidade de Conservação de Floresta Ombrófila Mista no Estado de Santa Catarina, Sul do Brasil. Realizamos procura ativa em sítios potencialmente utilizados para a reprodução das espécies nos meses de outubro de 2008 e março de 2009 . Registramos um total de 29 espécies, sendo 28 nativas e uma exótica, pertencentes a 16 gêneros e oito famílias. A elevada riqueza registrada, bem como a ocorrência de espécies relacionadas a ambientes íntegros, demonstra a importância do Parque Nacional das Araucárias para a conservação de anfíbios no Estado de Santa Catarina.

Palavras-chave: anfíbios, riqueza de espécies, conservação, Floresta de Araucária. 


\section{Introdução}

Nas últimas décadas, um número crescente de estudos e esforços para a conservação de anfíbios tem sido observado, em grande parte devido à constatação de declínio populacional e desaparecimento de muitas espécies em diversas regiões do mundo (Blaustein \& Wake 1990, Young et al. 2001, Kiesecker et al. 2001, Morrison \& Hero 2003). Outras razões responsáveis pelo aumento de estudos sobre conservação de anfíbios são a importância ecológica desse grupo na dinâmica trófica dos ecossistemas aquáticos e terrestres, a presença de princípios bioativos na pele e o potencial bioindicador de degradação ambiental (Seymour et al. 2001). Os anfíbios são especialmente vulneráveis à degradação dos ambientes naturais, pois possuem geralmente baixa mobilidade, restrições fisiológicas devido à pele altamente permeável e a dependência tanto de ambientes aquáticos como terrestres em diferentes fases do ciclo de vida (Alford \& Richards 1999).

O Brasil abriga mais de $10 \%$ da diversidade de anfíbios do mundo, mas tanto o conhecimento do status taxonômico quanto da distribuição geográfica da maioria das espécies é ainda bastante fragmentado (Silvano \& Segalla 2005), o que dificulta a compreensão sobre possíveis declínios e extinções (Heyer et al. 1988, Eterovick et al. 2005). Segundo a lista das espécies da fauna brasileira ameaçada de extinção (IBAMA 2003) e a lista internacional de espécies ameaçadas (IUCN 2010), a grande maioria dos anfíbios ameaçados de extinção no Brasil (93\%, $\mathrm{n}=14$ da lista brasileira e 82\%, $\mathrm{n}=24$ da lista internacional) ocorre exclusivamente na Mata Atlântica, a qual abriga alta diversidade e endemismo de espécies desse grupo (Haddad \& Prado 2005), ao mesmo tempo em que é um dos biomas com maior grau de destruição e níveis de ameaça (Myers et al. 2000, Ribeiro et al. 2009). A destruição dos habitats naturais é a principal ameaça à conservação de anfíbios na Mata Atlântica (Becker et al. 2007), assim como para a maioria das espécies dos diferentes grupos no mundo (Sala et al. 2000, Young et al. 2001).

Poucas espécies da Mata Atlântica encontram-se formalmente ameaçadas de extinção e muitas necessitam de mais dados para uma avaliação adequada (categoria 'dados insuficientes'), de acordo com o conhecimento atual (IBAMA 2003, IUCN 2010). Isso demonstra a falta de informações sobre elas e indica que ações urgentes e bem planejadas podem ser importantes para evitar a extinção de muitas espécies (Silva \& Casteleti 2003). Para subsidiar essas ações, são necessários esforços visando conhecer a diversidade de espécies dos diferentes grupos e a variação na composição de espécies destes grupos em diferentes áreas ou regiões (Rocha et al. 2004, Langhammer et al. 2007). Nesse sentido, inventários de fauna são importantes ferramentas, pois subsidiam avaliações e definições de estratégias e ações mais eficientes para a conservação e manejo da biodiversidade (Santos 2003).

Neste estudo, nós apresentamos uma lista de espécies e informações sobre o uso de ambientes, biologia reprodutiva e as principais ameaças aos anfíbios anuros ocorrentes no Parque Nacional das Araucárias, região Oeste do Estado de Santa Catarina, Sul do Brasil. A região Oeste de Santa Catarina é caracterizada pela intensa ocupação do solo e carece de conhecimento sobre a fauna dos poucos remanescentes de vegetação original. Somente dois estudos publicados de levantamento de fauna de anfíbios em áreas naturais (Lucas \& Fortes 2008, Hartmann et al. 2008) são conhecidos desta região.

\section{Material e Métodos}

\section{1. Área de estudo}

O estudo foi realizado no Parque Nacional das Araucárias, Unidade de Conservação (UC) de proteção integral, com 12.839 ha, localizada nos municípios de Ponte Serrada e Passos Maia, Oeste do Estado de Santa Catarina, Sul do Brasil (Figura 1). O clima da região é temperado, mesotérmico brando (média entre 10 e $15^{\circ} \mathrm{C}$ ), sem estação seca definida (IBGE 2002).

A região de estudo está inserida no bioma Mata Atlântica, e a fitofisionomia predominante é a Floresta Ombrófila Mista, a qual se caracteriza pela presença marcante de Araucaria angustifolia no estrato superior (IBGE 2008). A Floresta Ombrófila Mista cobria originalmente 42,5\% do Estado de Santa Catarina (Medeiros 2006), mas é pobremente representada por UC's de proteção integral nesse estado (Lucas 2008).

O Parque Nacional das Araucárias é um dos últimos remanescentes e a maior UC de Floresta Ombrófila Mista em Santa Catarina. Nessa área, são observadas formações florestais em aparentemente bom estado de conservação e baixa interferência antrópica; vegetação natural em diferentes estágios de sucessão; reflorestamento de espécies nativas (e.g. erva-mate) e exóticas (Pinus e Eucalyptus) e áreas abertas com plantios agrícolas e pastagens, abandonados ou recentemente manejados (Prochnow 2009). Próximo ao PN Araucárias existem remanescentes de campos naturais, os quais são utilizados como pastagem para a criação de gado.

As amostragens de anfíbios foram realizadas em seis sítios e concentradas nos ambientes naturais mais íntegros com possibilidade de acesso (Caratuva: $26,840^{\circ} \mathrm{S}, 51,988^{\circ} \mathrm{O}$; Rio do Mato: $26,813^{\circ} \mathrm{S}$, 51,903 ${ }^{\circ} \mathrm{O}$; Vila Adami: $26,827^{\circ} \mathrm{S}, 51,812^{\circ} \mathrm{O}$; Fazenda Marconstrói: $26,771^{\circ} \mathrm{S}, 51,955^{\circ} \mathrm{O}$; Fazenda Santa Fé: $26,700^{\circ} \mathrm{S}, 51,924^{\circ} \mathrm{O}$ e Fazenda Ameixeira: $26,756^{\circ} \mathrm{S}, 51,970^{\circ} \mathrm{O}$ ). Nesses sítios, diferentes ambientes propícios à ocorrência de anuros foram investigados, tais como i) trilhas no interior de mata com abundante folhiço, ii) córregos com fundo rochoso e/ou lodoso, iii) lagoas e banhados naturais, temporários e permanentes, em borda ou interior de mata, iv) lagoas artificiais e banhados em área aberta, e v) margens de rio com fundo rochoso (Figura 2).

Lagoas e banhados localizados em áreas abertas e na borda de mata nativa foram amostrados em todos os sítios (Figura 2a). As lagoas permanentes geralmente apresentavam abundante vegetação aquática flutuante e emergente, incluindo lagoas com toda a superfície coberta por plantas aquáticas da família Lemnaceae (e.g. Wolffia sp. Figura 2b). Essas lagoas, e também as temporárias localizadas no interior de mata ocorreram principalmente no Rio do Mato e Caratuva. As lagoas localizadas nas áreas abertas alteradas eram cercadas principalmente por gramíneas exóticas. Poças temporárias e permanentes nas margens de rio com fundo rochoso foram amostradas somente na Fazenda Santa Fé.

Nos sítios Caratuva e Fazenda Ameixeira, os riachos no interior de mata eram caracterizados pela presença marcante de xaxins (Dicksonia sellowiana e Nephelea setosa) nas margens, e abundante folhiço e troncos caídos no leito. Esses riachos apresentavam fundo lodoso e/ou rochoso. Em todos os sítios, com exceção da Fazenda Ameixeira e especialmente no Rio do Mato e Caratuva, além dos riachos, foram amostrados pequenos filetes d'água com largura inferior a $1 \mathrm{~m}$, localizados no interior e borda de mata nativa. Esses filetes d'água apresentavam fundo predominantemente lodoso com folhiço e vegetação arbustiva nas margens.

\section{Coleta e análise de dados}

A coleta de dados foi realizada em duas campanhas nos períodos de 18 a 27 de outubro de 2008 e 11 a 20 de março de 2009, durante a elaboração do Plano de Manejo da UC, totalizando um esforço amostral de aproximadamente 220 horas/homem. A amostragem foi baseada no método de levantamento em sítios reprodutivos (sensu Scott Jr \& Woodward 1994). As atividades de procura foram realizadas principalmente no período noturno, com auxílio de lanternas, onde todos os microhábitats visualmente acessíveis eram examinados. 
Iniciamos as atividades logo após o entardecer e encerramos após a diminuição da atividade de vocalização das espécies. Durante o dia, abrigos potenciais e corpos d'água eram examinados, em diferentes horários, em busca de espécies de hábito diurno, indivíduos em repouso e girinos. Para o registro das vocalizações utilizamos gravador Sony TCM 353-V e microfone direcional Yoga EM9600.

A identificação das espécies foi feita com base em bibliografia, consulta à especialistas e comparação com exemplares depositados em coleções científicas. Os exemplares coletados foram medidos com auxílio de paquímetro $(0,05 \mathrm{~mm})$ e depositados na Coleção Herpetológica da Universidade Federal de Minas Gerais (UFMG) e Coleção de Anfíbios da Universidade Comunitária da Região de Chapecó (Licença SISBIO nº 16954-1). A classificação taxonômica das espécies registradas seguiu as revisões taxonômicas recentes (e.g., Cassini et al. 2010, Frost 2010, Lavilla et al. 2010).

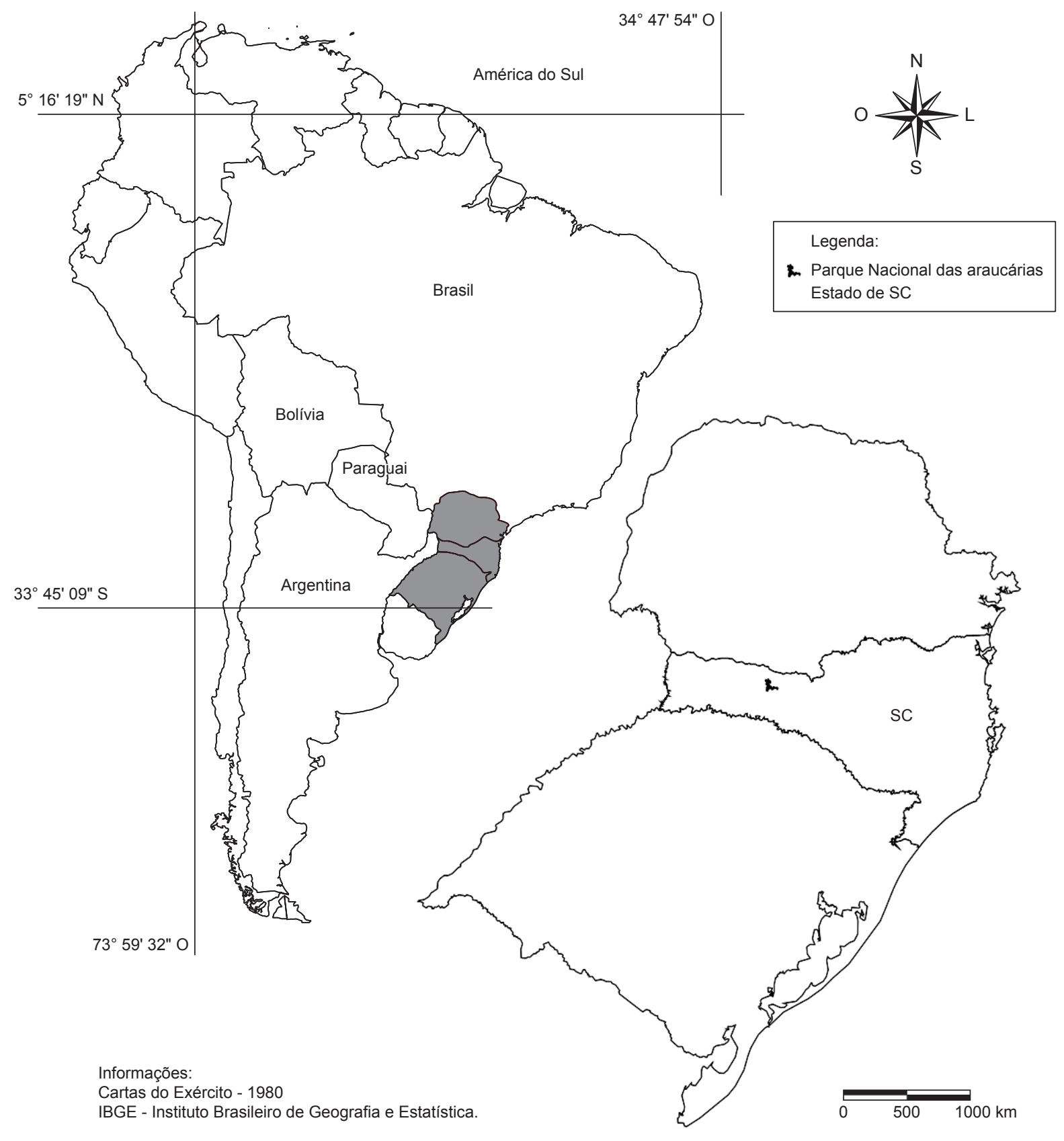

Informações sobre uso do ambiente e biologia reprodutiva das espécies foram registradas sempre que possível. O modo reprodutivo, o qual é baseado no local de deposição dos ovos e de desenvolvimento dos girinos, foi considerado de acordo com as classificações de Haddad \& Prado (2005) e Haddad et al. (2008) para os anfíbios da Mata Atlântica. Para esta classificação, utilizamos informações da literatura (Pombal \& Haddad 2005, Haddad et al. 2008, Armstrong \& Conte 2010) e observações de campo. Além das informações sobre as espécies, foram registradas potenciais ameaças à conservação dos anfíbios dentro e no entorno da UC. Para isso, para cada sítio amostrado e seus arredores foram registradas as influências antrópicas mais evidentes, como remoção da vegetação natural, presença de espécies exóticas da fauna e da flora, vestígios recentes da presença de animais domésticos e exóticos nos corpos d'água, presença de plantio agrícola, presença e proximidade de estradas e moradias. 
Curvas de acumulação de espécies foram geradas com auxílio do programa Estimates 7.5.0 (Colwell 2005), calculadas com os dados diários de coleta das duas campanhas de campo.

\section{Resultados e Discussão}

Registramos 29 espécies da ordem Anura, sendo 28 nativas e uma exótica, as quais pertencem a 16 gêneros e oito famílias (Tabela 1, Figura 3). Hylidae foi a família com maior número de espécies registradas $(\mathrm{n}=16,55 \%$, Tabela 1$)$. A família Ranidae foi representada pela espécie exótica Lithobates catesbeianus (rã-touro).

Apesar de próxima da estabilização, a curva de acumulação de espécies demonstrou que ainda não foram registradas todas as espécies de anuros possivelmente ocorrentes na área (Figura 4). De fato, além de outros possíveis registros, algumas espécies com ocorrência conhecida na região, como Leptodactylus fuscus, Leptodactylus mystacinus, Elachistocleis bicolor, Hypsiboas albopunctatus e Scinax aromothyella (Lucas \& Fortes 2008, Hartmann et al. 2008), não foram observadas no período de estudo. Mesmo assim, a riqueza registrada se aproxima da conhecida em outros remanescentes com Floresta Ombrófila Mista no sul do Brasil (Kwet \& Di-Bernardo 1999, São Francisco de Paula, RS: 36 espécies, Conte \& Machado 2005, Tijucas do Sul, PR: 23 espécies, Conte \& Rossa-Feres 2006, São José dos Pinhas, PR: 34 espécies, Conte \& Rossa-Feres 2007, Fazenda Rio Grande, PR: 32 espécies, Lucas \& Fortes 2008, Guatambu e Chapecó, SC: 29 espécies, Hartmann et al. 2008, Ipuaçú, SC: 21 espécies, Lingnau 2009, Lebon Régis, SC: 32 espécies), nos quais foram conduzidas amostragens regulares ao longo de um ano ou mais.

Foi possível registrar pelo menos oito modos reprodutivos na taxocenose estudada, os quais representam $29 \%$ dos modos reprodutivos conhecidos para a Mata Atlântica (Tabela 1). A maioria das espécies se reproduz em corpos d'água lênticos (86\%, Modos 1, 4, 11, 23, 24 e 30), nos quais girinos exotróficos se desenvolvem (Tabela 1). O Modo 1 foi o mais comum, representado por 15 espécies (50\%), seguido do Modo $11(\mathrm{n}=4,13 \%)$ e o Modo $2(\mathrm{n}=2,6 \%)$. Os Modos 4, 5, 23, 24, 25 e 30 foram representados por somente uma espécie (Tabela 1). Cinco espécies (17\%) são relacionadas a modos reprodutivos considerados tipicamente florestais (Modos 2, 5, 23 e 25), enquanto as demais apresentam modos reprodutivos associados tanto a áreas abertas quanto florestadas (Haddad \& Prado 2005, Tabela 1).

Vinte e quatro espécies (83\%) utilizaram áreas com influência antrópica (abertas e/ou borda de mata), mas algumas ocorreram exclusivamente ou predominantemente ambientes florestais mais íntegros na área de estudo, como Ischnocnema henselii, Rhinella henseli, Proceratophrys bigibbosa, Vitreorana uranoscopa, Trachycephalus dibernardoi e Physalaemus cf. lateristriga (Tabela 1). Vitreorana uranoscopa e Sphaenorhynchus surdus são considerados ameaçados de extinção (categoria 'vulnerável') no Estado do Rio Grande do Sul (Marques et al. 2002). Vitreorana uranoscopa é também classificada na categoria 'dados insuficientes' na lista vermelha da fauna ameaçada de extinção do Estado do Paraná (Mikich \& Bernils 2004) e Proceratophrys bigibbosa consta na categoria 'quase ameaçada' na lista internacional de espécies ameaçadas de extinção (IUCN 2010).

Hypsiboas leptolineatus e Sphaenorhynchus surdus são endêmicas da Floresta Ombrófila Mista (Conte 2010), e outras espécies, como Rhinella henseli e Trachycephalus dibernardoi possuem grande parte da área de ocupação nesse ecossistema. No entanto, ainda pouco se sabe sobre a distribuição geográfica, ecologia e taxonomia das espécies ocorrentes na Floresta Ombrófila Mista (Lucas \& Fortes 2008, Conte 2010, Conte et al. 2010) e tanto a diversidade como o grau de endemismo de anfíbios deve estar subestimado (Conte
2010). A escassez de conhecimento, aliada a intensa fragmentação e deterioração da Floresta Ombrófila Mista, a qual é considerada uma das fitofisionomias mais ameaçadas do bioma Mata Atlântica (Hirota 2003, Ribeiro et al. 2009), é uma séria ameaça à conservação das espécies, especialmente daquelas endêmicas.

Detectamos diversas ameaças potenciais à conservação dos anuros no entorno e no interior do Parque Nacional das Araucárias, como a presença de espécies exóticas e domésticas da fauna e plantios agrícolas com uso de agrotóxicos. A mais marcante é a presença de espécies exóticas e domésticas, como javalis, bovinos, equinos, suínos domésticos e a rã-touro; os quais são encontrados com elevada frequência no interior da área, inclusive nos ambientes mais íntegros. Esses animais, especialmente javalis, alteram intensamente os corpos d'água lóticos e lênticos utilizados para a reprodução dos anuros, compactando o solo e interferindo na dinâmica da água e da vegetação das margens onde são estabelecidos os sítios de canto e desova. A rã-touro, que também representa uma ameaça às espécies nativas de anuros, possui elevado potencial de competição e predação (Alves et al. 2008).

Considerando a elevada riqueza registrada neste estudo preliminar, a qual representa cerca de $20 \%$ das espécies de anuros conhecidas no Estado de Santa Catarina (Lucas 2008), bem como a ocorrência de espécies exclusivas da Floresta Ombrófila Mista e de ambientes íntegros e as potenciais ameaças à anurofauna, é desejável a realização de estudos sistemáticos mais detalhados e de longo prazo na área. Tais estudos poderão avaliar melhor a diversidade de espécies e o impacto das ameaças sobre os anuros, bem como possíveis medidas de manejo que possam apoiar o planejamento da UC. Nossos resultados sustentam a importância do Parque Nacional das Araucárias como área de grande importância para a conservação dos anfíbios da Floresta Ombrófila Mista e de toda Mata Atlântica, um dos biomas mais ameaçados do mundo.

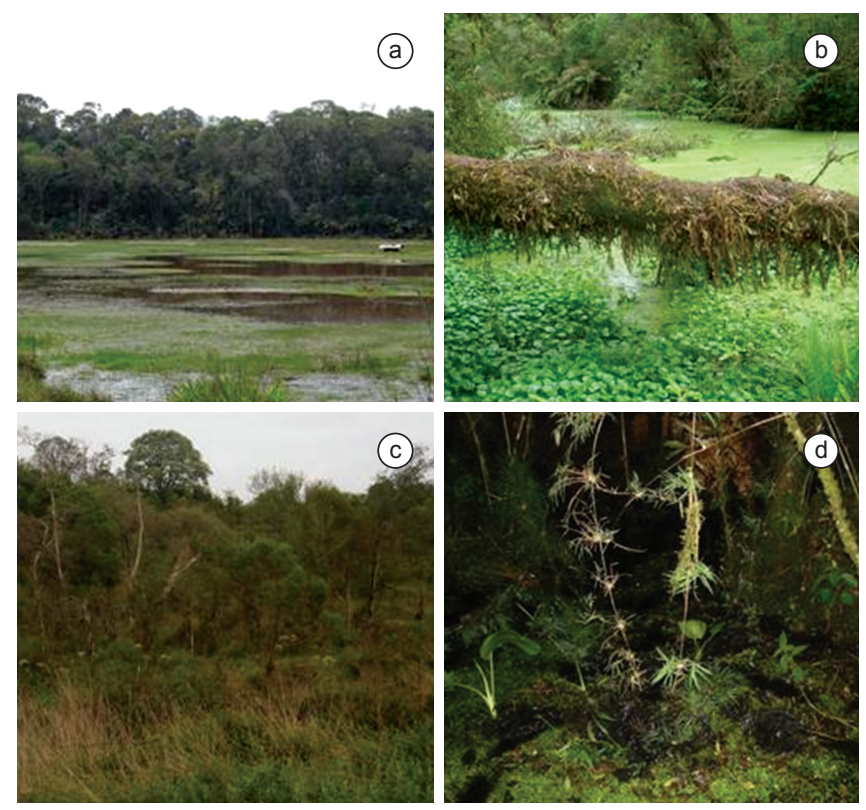

Figura 2. Alguns ambientes amostrados no Parque Nacional das Araucárias, Estado de Santa Catarina, Brasil. a) Lagoa permanente na borda de mata nativa; b) lagoa permanente no interior de mata nativa; c) banhado em área de vegetação em regeneração; e d) riacho permanente no interior de mata nativa.

Figure 2. Some of the sites sampled in Parque Nacional das Araucárias, state of Santa Catarina, southern Brazil. a) Permanent pond on the edge of the native forest; b) permanent pond inside the native forest; c) swamp in regenerating area; and d) permanent stream inside the native forest. 

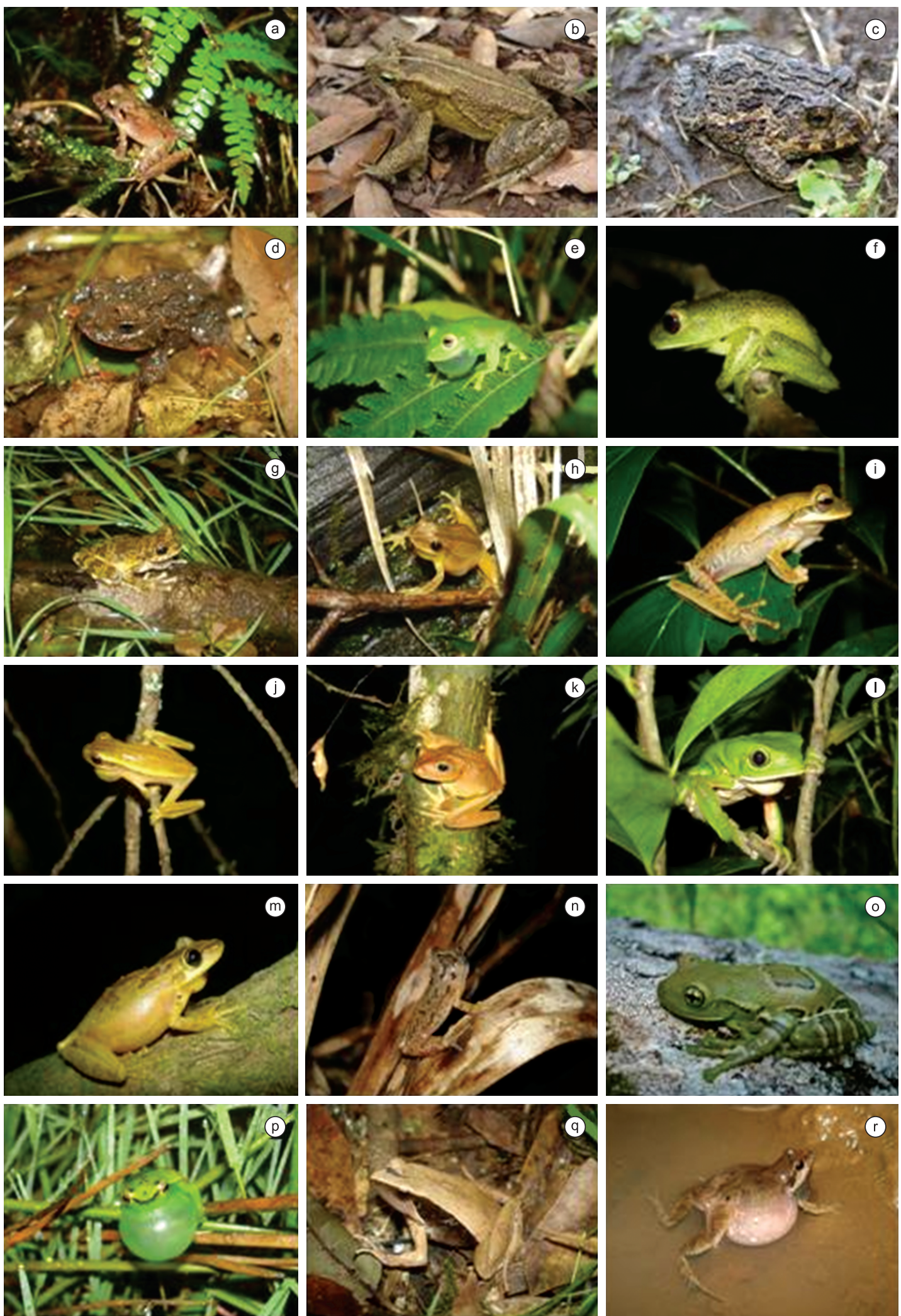

Figura 3. Anfíbios anuros registrados no Parque Nacional das Araucárias, Estado Santa Catarina, Sul do Brasil. a) Ischnocnema henselii; b) Rhinella henseli; c) Odontophrynus americanus; d) Proceratophrys bigibbosa; e) Vitreorana uranoscopa; f) Aplastodiscus perviridis; g) Dendropsophus nahdereri; h) Dendropsophus minutus; i) Hypsiboas bischoffi; j) Hypsiboas leptolineatus; k) Hypsiboas faber; 1) Phyllomedusa tetraploidea; m) Scinax perereca; n) Scinax uruguayus; o) Trachycephalus dibernardoi; p) Sphaenorhynchus surdus; q) Physalaemus cf. lateristriga; e r) Physalaemus cuvieri. Fotos: E.M. Lucas.

Figure 3. Anuran species recorded in Parque Nacional das Araucárias, Santa Catarina State, Southern Brazil. a) Ischnocnema henselii; b) Rhinella henseli; c) Odontophrynus americanus; d) Proceratophrys bigibbosa; e) Vitreorana uranoscopa; f) Aplastodiscus perviridis; g) Dendropsophus nahdereri; h) Dendropsophus minutus; i) Hypsiboas bischoffi; j) Hypsiboas leptolineatus; k) Hypsiboas faber; 1) Phyllomedusa tetraploidea; m) Scinax perereca; n) Scinax uruguayus; o) Trachycephalus dibernardoi; p) Sphaenorhynchus surdus; q) Physalaemus cf. lateristriga; and r) Physalaemus cuvieri. Photos: E.M. Lucas. 
Tabela 1. Espécies de anuros, ambiente de registro, comprimento rostro-cloacal de machos (CRC; média \pm desvio padrão; $\mathrm{n}=$ número de indivíduos; amplitude) e modo reprodutivo, registrados em outubro de 2008 e março de 2009 no Parque Nacional das Araucárias, Estado de Santa Catarina, sul do Brasil. IM: interior de mata nativa, com baixa interferência antrópica; $\mathrm{BO}$ = borda de mata ou fragmentos de vegetação natural em diferentes estágios sucessionais; $\mathrm{AA}=$ áreas abertas ou de reflorestamento com exóticas, com acentuada interferência antrópica. MR = modo reprodutivo, conforme Haddad \& Prado (2005) e Haddad et al. (2008). 1) ovos e girinos exotróficos em água parada; 2) ovos e girinos exotróficos em água corrente; 4) desova e desenvolvimento inicial dos girinos em piscinas de barro construídas ou naturais; após a inundação os girinos exotróficos se desenvolvem em poças ou riachos; 5) ovos e estágios iniciais em ninhos subterrâneos construídos, após o transbordamento, girinos exotróficos em poças ou riachos; 11) ovos em ninhos de espuma flutuantes e girinos exotróficos em poças; 23) desenvolvimento direto de ovos terrestres; 24) ovos arborícolas, com eclosão de girinos exotróficos que caem das folhas em água parada; 25) ovos arborícolas que eclodem em girinos exotróficos que caem das folhas em água corrente; e 30) ovos em ninhos de espuma e desenvolvimento inicial dos girinos em ninhos subterrâneos construídos, após o transbordamento, girinos exotróficos em poças.

Table 1. Anuran species, habitat, snout-vent length of males (mean \pm standard deviation; $\mathrm{n}=$ number of individuals; range) and reproductive mode, recorded in October 2008 and March 2009 in Parque Nacional das Araucárias, Santa Catarina State, southern Brazil. IM: inside native forest with low human disturbance; $\mathrm{BO}=$ edge of forest or inside small recovering fragments; $\mathrm{AA}=$ open areas and stands of exotic species with high human disturbance. MR $=$ reproductive mode, according to Haddad \& Prado (2005) and Haddad et al. (2008). 1) eggs and exotrophic tadpoles in still water; 2) eggs and exotrophic tadpoles in flowing water; 4) eggs and early larval stages in natural or constructed basins; subsequent to flooding, exotrophic tadpoles in ponds or streams; 5) eggs and early larval stages in subterranean constructed nests; subsequent to flooding, exotrophic tadpoles in ponds or streams; 11) foam nest floating on pond; exotrophic tadpoles in ponds; 23) direct development of terrestrial eggs; 24) arboreal eggs hatch into exotrophic tadpoles that drop in lentic water; 25) arboreal eggs hatch into exotrophic tadpoles that drop in flowing water; and 30) foam nest with eggs and early larval stages in subterranean constructed nests; subsequent to flooding, exotrophic tadpoles in ponds.

Familia/Especie

Família/Espécie

Brachycephalidae

Ischnocnema henselii (Peters, 1870)

Bufonidae

Melanophryniscus sp. (grupo tumifrons)

Rhinella henseli (Lutz, 1934)

Rhinella icterica (Spix, 1824)

Centrolenidae

Vitreorana uranoscopa (Müller, 1924)

Cycloramphidae

Odontophrynus americanus (Duméril \& Bibron, 1841)

Proceratophrys bigibbosa (Peters, 1872)

Hylidae

Aplastodiscus perviridis Lutz, 1950

Dendropsophus minutus (Peters, 1872)

Dendropsophus nahdereri (Lutz \& Bokermann, 1963)

Hypsiboas bischoffi (Boulenger, 1887)

Hypsiboas curupi Garcia, Faivovich \& Haddad, 2007

Hypsiboas faber (Wied-Neuwied, 1821)

Hypsiboas leptolineatus (Braun \& Braun, 1977)

Phyllomedusa tetraploidea Pombal \& Haddad, 1992

Scinax berthae (Barrio, 1962)

Scinax fuscovarius (Lutz, 1925)

Scinax cf. granulatus (Peters, 1871)

Scinax perereca Pombal, Haddad \& Kasahara, 1995

\section{IM BO AA}

$\mathrm{CRC}(\mathbf{m m})$

MR

$\mathrm{x} \quad-\quad(\mathrm{n}=3 ; 25,0-25,6)$
$\quad$

$(\mathrm{n}=3 ; 25,0-25,6)$$$
\mathrm{X}
$$$$
-
$$$$
22,7 \pm 1,3
$$$$
(\mathrm{n}=5 ; 21,3-24,5)
$$$$
\text { - } \quad-\quad x
$$$$
\mathrm{x} \quad \mathrm{X} \quad-
$$$$
31,6 \pm 5,7
$$$$
\text { (n }=2 ; 27,6-35,6)
$$$$
\text { - x - }
$$$$
41,0 \pm 1,8
$$$$
(\mathrm{n}=4 ; 39,3-43,6)
$$$$
23,0 \pm 0,9
$$$$
\text { (n }=8 ; 21,7-24,5)
$$$$
42,3 \pm 3,2
$$$$
(\mathrm{n}=5 ; 36,8-44,8)
$$$$
41,9 \pm 0,6
$$$$
(\mathrm{n}=2 ; 41,4-42,4)
$$$$
39,9
$$$$
(\mathrm{n}=1)
$$$$
92,3 \pm 6,2
$$$$
(\mathrm{n}=2 ; 88,0-96,7)
$$$$
27,2 \pm 0,8
$$$$
1
$$$$
1
$$

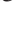

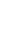

5$$
\text { (n }=3 ; 26,6-28,2)
$$$$
60,0 \pm 4,1
$$$$
\text { ( } \mathrm{n}=5 ; 54,1-65,3)
$$$$
19,2 \pm 0,6
$$$$
(\mathrm{n}=6 ; 18,6-20,2)
$$$$
44,3 \pm 0,6
$$$$
\text { ( } \mathrm{n}=4 ; 43,6-45,1)
$$$$
32,7 \pm 1,7
$$$$
(\mathrm{n}=6 ; 31,1-35,5)
$$$$
35,9 \pm 1,7
$$

$(\mathrm{n}=18 ; 33,1-38,0)$ 
Tabela 1. Continuação...

\begin{tabular}{|c|c|c|c|c|c|}
\hline Família/Espécie & IM & BO & AA & $\mathrm{CRC}(\mathbf{m m})$ & MR \\
\hline Scinax squalirostris (Lutz, 1925) & - & - & $\mathrm{x}$ & $\begin{array}{c}24,1 \\
(n=1)\end{array}$ & 1 \\
\hline Scinax uruguayus (Schmidt, 1944) & - & $\mathrm{x}$ & $\mathrm{x}$ & $\begin{array}{c}24,3 \pm 1,1 \\
(\mathrm{n}=4 ; 24,1-25,7)\end{array}$ & 1 \\
\hline Sphaenorhynchus surdus (Cochran, 1953) & - & $\mathrm{x}$ & $\mathrm{x}$ & $\begin{array}{c}26,8 \pm 2,5 \\
(\mathrm{n}=3 ; 24,5-29,5)\end{array}$ & 1 \\
\hline Trachycephalus dibernardoi Kwet \& Solé, 2008 & $\mathrm{x}$ & - & - & $\begin{array}{c}56,7 \\
(n=1)\end{array}$ & - \\
\hline \multicolumn{6}{|l|}{ Leiuperidae } \\
\hline Physalaemus aff. gracilis & - & $\mathrm{x}$ & $\mathrm{x}$ & $\begin{array}{c}24,6 \pm 3,7 \\
(\mathrm{n}=2 ; 22,0-27,2)\end{array}$ & 11 \\
\hline Physalaemus cuvieri Fitzinger, 1826 & - & $\mathrm{x}$ & $\mathrm{x}$ & $\begin{array}{c}28,2 \pm 2,0 \\
(\mathrm{n}=3 ; 26,4-30,4)\end{array}$ & 11 \\
\hline Physalaemus cf. lateristriga & $\mathrm{x}$ & - & - & - & 11 \\
\hline \multicolumn{6}{|l|}{ Leptodactylidae } \\
\hline Leptodactylus latrans (Steffen, 1815) & - & - & $\mathrm{x}$ & $\begin{array}{c}84,6 \\
(\mathrm{n}=1)\end{array}$ & 11 \\
\hline Leptodactylus plaumanni Ahl, 1936 & - & - & $\mathrm{x}$ & - & 30 \\
\hline \multicolumn{6}{|l|}{ Ranidae } \\
\hline Lithobates catesbeianus (Shaw, 1802) & - & - & $\mathrm{x}$ & - & 1 \\
\hline Total & 9 & 16 & 17 & & 8 \\
\hline
\end{tabular}

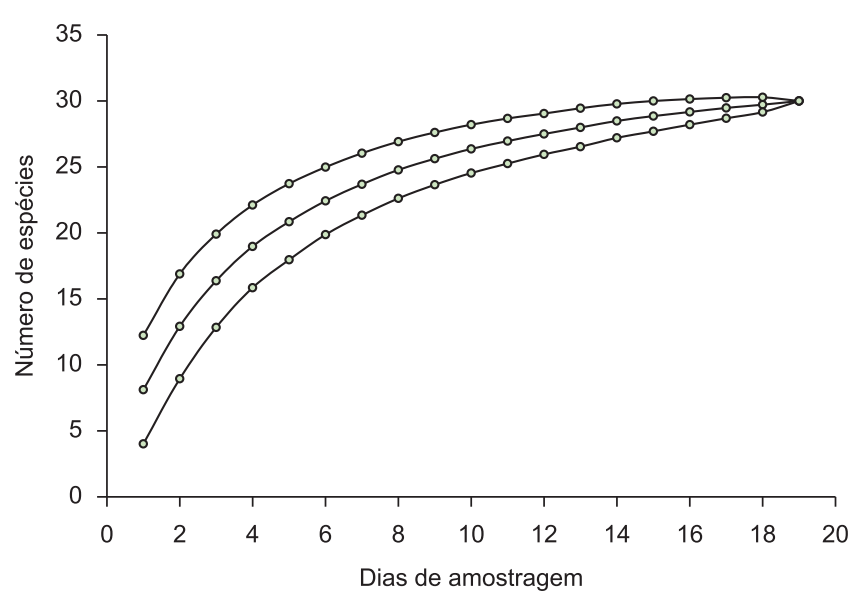

Figura 4. Curvas de acumulação de espécies, calculadas com os dados obtidos nos períodos de 18 a 26 de outubro de 2008 e 11 a 20 de março de 2009, no Parque Nacional das Araucárias, Estado de Santa Catarina, Brasil. Os pontos correspondem à média e desvio padrão das 1.000 curvas geradas com ordem aleatória de amostras.

Figure 4. Species accumulation curves, based on data collected from October 18 to $26^{\text {th }} 2008$ and March 11 to $20^{\text {th }} 2009$, in Parque Nacional das Araucárias, Santa Catarina State, Brazil. Circles represent the mean of 1000 points generated with randomly ordered samples.

\section{Agradecimentos}

Agradecemos a APREMAVI - Associação de Preservação do Meio Ambiente e da Vida e ao diretor do Parque Nacional das Araucárias, Juliano de Oliveira, pela organização e apoio logístico, a ACAPRENA - Associação Catarinense de Preservação da Natureza e demais apoiadores do Plano de Manejo (Cooperação República Federativa do Brasil e República Federal da Alemanha, PDA Mata Atlântica, KFW, GTZ, Ministério do Meio Ambiente, Banco do Brasil, The Nature Conservancy) pelo auxílio logístico e financeiro;
ICMBio pela licença de coleta; Paulo C. A. Garcia pelo auxílio nas identificações; Edilaine Dick, Marcos A. Danielli, Adrian Rupp, Cintia Gruener, Bruna Savi, Bruna Turra, Erikcsen Raimundi, Jean Bernardon, Jaqueline Pesenti, Carlize Pasquali e Franciele Dias pela companhia e auxílio nos trabalhos de campo; Cristina O. Gridi-Papp pelo auxílio no inglês e a dois revisores anônimos pelas contribuições na versão preliminar do trabalho.

\section{Referências Bibliográficas}

ALFORD, R.A. \& RICHARDS, S.J. 1999. Global amphibian declines: a problem in applied ecology. Annu. Rev. Ecol. Syst. 30:133-165.

ALVES, F.C., BRANCO, A., LUCAS, E.M. \& FORTES, V.B. 2008. Ocorrência da espécie exótica Lithobates catesbeianus (rã-touro) em ambientes naturais nos municípios de Chapecó e Guatambu, Santa Catarina, Brasil. Acta Amb. Catar. 5(1-2):35-42.

ARMSTRONG, C.G. \& CONTE, C.E. 2010. Taxocenose de anuros (Amphibia: Anura) em uma area de Floresta Ombrófila Densa no sul do Brasil. Biota Neotrop. 10(1): http://www.biotaneotropica.org.br/v10n1/ en/abstract?article+bn00610012010 (último acesso em 22/07/2010).

BECKER, C.G., FONSECA, C.R., HADDAD, C.F.B., BATISTA, R.F. \& PRADO, P.I. 2007. Habitat split and the global decline of amphibians. Science 318:1775-1777.

BLAUSTEIN, A.R. \& WAKE, D.B. 1990. Declining amphibian populations - a global phenomenon. Trends Ecol. Evol. 5:203-204.

CASSINI, C.S., CRUZ, C.A.G. \& CARAMASCHI, U. 2010. Taxonomic review of Physalaemus olfersii (Lichtenstein \& Martens, 1856) with revalidation of Physalaemus lateristriga (Steindachner, 1864) and description of two new related species (Anura: Leiuperidae). Zootaxa 2491:1-33.

COLWELL, R.K. 2005. Estimates: statistical estimation of species richness and shared species from samples. Version 7.5. User's Guide and application published at: http://purl.oclc.org/estimates.

CONTE, C.E. 2010. Diversidade de anfíbios da Floresta com Araucária. Tese de doutorado, Universidade Estadual Paulista Júlio de Mesquita Filho, São José do Rio Preto.

CONTE, C.E. \& MACHADO, R.A. 2005. Riqueza de espécies e distribuição espacial e temporal em comunidade de anuros (Amphibia, Anura) em uma localidade de Tijucas do Sul, Paraná, Brasil. Rev. Bras. Zool. 22(4):940-948.

CONTE, C.E. \& ROSSA-FERES, D.C. 2006. Diversidade e ocorrência temporal da anurofauna (Amphibia, Anura) em São José dos Pinhais, Paraná, Brasil. Rev. Bras. Zool. 23(1):162-175. 
CONTE, C.E. \& ROSSA-FERES, D.C. 2007. Riqueza e distribuição espaçotemporal de anuros em um remanescente de Floresta de Araucária no sudeste do Paraná. Rev. Bras. Zool. 24(4):1025-1037.

CONTE, C.E., NOMURA, F., MACHADO, R.A., KWET, A., LINGNAU, R. \& ROSSA-FERES, D.C. 2010. Novos registros na distribuição geográfica de anuros na Floresta com Araucária e consideração sobre suas vocalizações. Biota Neotrop. 10(2): http://www.biotaneotropica.org.br/ v10n2/pt/fullpaper?bn01110022010+pt (último acesso em 18/11/2010).

ETEROVICK, P.C., CARNAVAL, A.C.O.Q., BORGES-NOJOSA, D.V., SILVANO, D.L., SEGALLA, M.V. \& SAZIMA, I. 2005. Amphibiam declines in Brazil: an overview. Biotropica 37(2):166-179.

FROST, D.R. 2010. Amphibian species of the world: an online reference. Version 5.4 (8 April, 2010). Electronic Database accessible at http:// research.amnh.org/vz/herpetology/amphibia/. American Museum of Natural History, New York, USA

HADDAD, C.F.B. \& PRADO, C.P.A. 2005. Reproductive modes in frogs and their unexpected diversity in the Atlantic Forest of Brazil. BioScience 55(3):207-217.

HADDAD, C.F.B., TOLEDO, L.F. \& PRADO, C.P.A. 2008. Anfíbios da Mata Atlântica: guia dos anfíbios anuros da Mata Atlântica. Ed. Neotropica, São Paulo.

HARTMANN, M.T., Garcia, P.C.A., Giasson, L.O.M. \& Hartmann, P.A. 2008. Anfíbios. In A fauna das áreas de influência da Usina Hidrelétrica Quebra Queixo (J.J. Cherem \& M. Kammers, orgs.). Habilis, Erechim, RS, p.89-107.

HEYER, W.R., RAND, A.S., CRUZ, C.A.G. \& PEIXOTO, O.L. 1988. Decimations, extinctions, and colonizations of frog populations in southeast Brazil and their evolutionary implications. Biotropica 20(3):230-235.

HIROTA, M.M. 2003. Monitoring the Brazilian Atlantic Forest Cover. In The Atlantic Forest of South America: biodiversity status, threats, and outlook (C. Galindo-Leal \& I.G. Câmara, eds.). Center for Applied Biodiversity Science and Island Press, Washington, D.C. p. 60-65.

INSTITUTO BRASILEIRO DE GEOGRAFIA E ESTATÍSTICA - IBGE. 2002. Mapa de clima do Brasil. Departamento de Recursos Naturais e Estudos Ambientais. ftp://geoftp.ibge.gov.br/mapas/tematicos/mapas_ murais/clima.pdf (último acesso em 03/08/2009).

INSTITUTO BRASILEIRO DE GEOGRAFIA E ESTATÍSTICA - IBGE. 2008. Mata Atlântica: mapa da área de aplicação da lei no 11.428 de 2006. Caracterização geral dos tipos de vegetação. Ministério do Planejamento, Orçamento e Gestão; Ministério do Meio Ambiente.

INSTITUTO BRASILEIRO DO MEIO AMBIENTE E DOS RECURSOS NATURAIS RENOVÁVEIS - IBAMA. 2003. Lista das espécies da fauna ameaçada de extinção. Instrução Normativa n 3, de 27 de maio de 2003. IBAMA; Ministério do Meio Ambiente. Brasília.

INTERNATIONAL UNION FOR CONSERVATION OF NATURE - IUCN. 2010. IUCN red list of threatened species. Version 2010.4. www. iucnredlist.org (último acesso em 04/11/2010).

KIESECKER, J.M., BLAUSTEIN, A.R. \& BELDEN, L.K. 2001. Complex causes of amphibian population declines. Nature 410:681-684.

KWET, A. \& DI-BERNARDO, M. 1999. Pró-mata-anfíbios. Amphibien. Amphibians. EDIPUCRS, Porto Alegre.

LANGHAMMER, P.F., BAKARR, M.I., BENNUM, L.A., BROOKS, T.M., CLAY, R.P., DARWALL, W., DE SILVA, N., EDGAR, G.J., EKEN, G., FISHPOOL, L.D.C., FONSECA, G.A.B., FOSTER, M.N., KNOX, D.H., MATIKU, P., RADFORD, E.A., RODRIGUES, A.S.L., SALAMAN, P., SECHREST, W. \& TORDOFF, A.W. 2007. Identification and gap analysis of key biodiversity areas: targets for comprehensive protected area systems. Gland, Switzerland: IUCN.

LAVILLA, E.O., LANGONE, J.A., CARAMASCHI, U., HEYER, W.R. \& DE SÁ, R. 2010. The identification of Rana ocellata Linnaeus, 1758. Nomenclatural impact on the species currently known as Leptodactylus ocellatus (Leptodactylidae) and Osteopilus brunneus (Gosse, 1851) (Hylidae). Zootaxa 2346:1-16.

LINGNAU, R. 2009. Distribuição temporal, atividade reprodutiva e vocalizações em uma assembléia de anfíbios anuros de uma Floresta Ombrófila Mista em Santa Catarina, sul do Brasil. Tese de doutorado, Pontifícia Universidade Católica do Rio Grande do Sul, Porto Alegre.
LUCAS, E.M. 2008. Diversidade e conservação de anfíbios anuros no Estado de Santa Catarina, Brasil. Tese de doutorado, Universidade de São Paulo, São Paulo.

LUCAS, E.M. \& FORTES, V.B. 2008. Frog diversity in the Floresta Nacional de Chapecó, Atlantic Forest of Southern Brazil. Biota Neotrop. 8(3): http:// www.biotaneotropica.org.br/v8n3/pt/abstract?article+bn00508032008 (último acesso $\mathrm{m}$ 22/07/2010).

MARQUES, A.A.B., FONTANA, C.S., VÉLEZ, E., BENCKE, G.A., SCHNEIDER, M. \& REIS, R.E. 2002. Lista das espécies da fauna ameaçadas de extinção no Rio Grande do Sul. Decreto n 41.672 , de 10 de junho de 2002. Porto Alegre: FZB/MCT-PUCRS/PANGEA.

MEDEIROS, J.D. 2006. Os estados da Mata Atlântica: Santa Catarina. In Mata Atlântica: uma rede pela floresta (M. Campanili \& M. Prochnow, orgs.). Brasília: RMA, p.45-57.

MIKICH, S.B. \& BÉRNILS, R.S. 2004. Livro vermelho da fauna ameaçada no Estado do Paraná. Governo do Paraná, Curitiba. http://www.pr.gov. br/iap (último acesso em 24/11/2009).

MORRISON, C. \& HERO, J.M. 2003. Geographic variation in life-history characteristics of amphibians: a review. J. Anim. Ecol. 72(2):270-279.

MYERS, N., MITTERMEIER, R.A., MITTERMEIER, C.G., FONSECA, G.A.B. \& KENT, J. 2000. Biodiversity hotspots for conservation priorities. Nature 403(6772):853-858.

POMBAL Jr, J.P. \& HADDAD, C.F.B. 2005. Estratégias e modos reprodutivos de anuros (Amphibia) em uma poça permanente na Serra de Paranapiacaba, Sudeste do Brasil. Pap. Avulsos de Zool. 45(15):201-213.

PROCHNOW, M. (org.). 2009. O Parque Nacional das Araucárias e a Estação Ecológica da Mata Preta: unidades de conservação da mata atlântica. Apremavi, Rio do Sul.

RIBEIRO, M.C., METZGER, J.P., MARTENSEN, A.C., PONZONI, F.J. \& HIROTA, M.M. 2009. Brazilian Atlantic forest: how much is left and how is the remaining forest distributed? Implications for conservation. Biolog. Conserv. 142:1141-1153.

ROCHA, C.F.D., BERGALLO, H.G., POMBAL Jr., J.P., GEISE, L., VAN SLUYS, M., FERNANDES, R. \& CARAMASCHI, U. 2004. Fauna de anfíbios, répteis e mamíferos do Estado do Rio de Janeiro, sudeste do Brasil. Publ. Avul. Mus. Nac. Rio de Janeiro 104:3-23.

SALA, O.E., CHAPIN, F.S., ARMESTO, J.J., BERLOW, E., BLOOMFIELD, J., DIRZO, R., HUBER-SANWALD, E., HUENNEKE, L.F., JACKSON, R.B., KINZIG, A., LEEMANS, R., LODGE, D.M., MOONEY, H.A., OESTERHELD, M., POFF, N.L., SYKES, M.T., WALKER, B.H., WALKER, M. \& WALL, D.H. 2000. Global biodiversity scenarios for the year 2100. Science 287:1770-1774.

SANTOS, A.J. 2003. Estimativas de riqueza em espécies. In Métodos de estudos em biologia da conservação \& manejo da vida silvestre (L. Cullen Jr., C. Valladares-Padua \& R. Rudran, orgs). Curitiba: Ed. da UFPR; Fundação o Boticário de Proteção à Natureza, p.19-41.

SCOTT Jr, N.J. \& WOODWARD, B.D. 1994. Revielamentos de lugares de reprodución. In Measuring and monitoring biological diversity - standard methods for amphibians (W.R. Heyer, M.A. Donnelly, R.W. McDiarmid, L.A.C. Hayek \& M.S. Foster, eds.). Smithsonian Institution Press, Washington, p.113-120.

SEYMOUR, C.L., DE KLERK, H.M., CHANNING, A. \& CROWE, T.M. 2001. The biogeography of the Anura of sub-equatorial Africa and the prioritization of areas for their conservation. Biodiversity Conserv. 10:2045-2076.

SILVA, J.M.C. \& CASTELETI, C.H.M. 2003. Status of the biodiversity of the Atlantic Forest of Brazil. In The Atlantic Forest of South America: biodiversity status, threats, and outlook. (C. Galindo-Leal, \& I.G. Câmara, eds.). Center for Applied Biodiversity Science and Island Press, Washington, D.C., p.43-59.

SILVANO, D.L. \& SEGALLA, M.V. 2005. Conservação de anfíbios no Brasil. Megadiversidade 1(1):79-86.

YOUNG, B., LIPS, K.R., REHACER, J.K., IBÁÑEZ, R., SALAS, A.W., CEDEÑO, J.R., COLOMA, L.A., RON, S., LA MARCA, E., MEYER, J.R., MUÑZ, A., BOLAÑOS, F., CHAVES, G. \& ROMO, D. 2001. Population declines and priorities for amphibian conservation in Latin America. Conserv. Biol. 15:1213-1223. 